\title{
Comparison over the Nuclear Analysis of the HCLL blanket for the European DEMO
}

\author{
Jean-Charles Jaboulay ${ }^{\mathrm{a}}$, Giacomo Aiello ${ }^{\mathrm{a}}$, Julien Aubert ${ }^{\mathrm{a}}$, Rosaria Villari ${ }^{\mathrm{b}}$, Ulrich Fischer ${ }^{\mathrm{c}}$ \\ ${ }^{a}$ CEA-Saclay, DEN, DM2S, F-91191 Gif-sur-Yvette, France \\ ${ }^{b}$ ENEA, UTFUS-TECN, Via E. Fermi 4, 00044 Frascati (Rome), Italy \\ ${ }^{c}$ Karlsruhe Institute of Technology, 76344 Eggenstein-Leopoldshafen, Karlsruhe, Germany
}

\begin{abstract}
This paper presents the comparison over the nuclear analysis of the European DEMO with HCLL blanket carried out with the TRIPOLI- $4{ }^{\circledR}$ Monte Carlo code and the JEFF-3.1.1 nuclear data library and with the MCNP5 Monte Carlo code and the FENDL-2.1 nuclear data library. The MCNP5 analysis was conducted firstly by ENEA with a detailed 3D model describing all the HCLL blanket internal structures. This MCNP5 model was converted into TRIPOLI- $4^{\circledR}$ representation for performing the nuclear analysis at CEA with the objective to demonstrate consistency between both analyses. A very good agreement was obtained for all of the relevant nuclear responses (neutron wall loading, tritium breeding ratio, nuclear heating, neutron flux distribution, etc.), validating CEA's nuclear analysis approach, based on TRIPOLI- $4^{\circledR}$ Monte Carlo code and JEFF-3.1.1 nuclear data library, for the European DEMO.
\end{abstract}

Keywords: DEMO, neutronics, blanket, HCLL, tritium breeding, nuclear heating, TRIPOLI-4 ${ }^{\circledR}$

\section{Introduction}

The EUROfusion Consortium [1] develops a conceptual design of a fusion power demonstrator (DEMO) in the framework of the European "Horizon 2020" innovation and research programme [2]. Key issues for DEMO are tritium self-sufficiency and heat removal for conversion into electricity. These functions are fulfilled by the breeding blankets surrounding the plasma chamber.

Within the Breeder Blanket project (WPBB) of EUROfusion's Power Plant Physics and Technology (PPPT) programme [3], CEA is in charge of the design of the helium cooled lithium lead (HCLL) blanket [4] for DEMO including the nuclear analyses. In WPBB's framework three other blanket concepts are respectively studied by KIT, ENEA and CIEMAT: the helium cooled pebble bed (HCPB), the water cooled lithium lead (WCLL) and the dual coolant lithium lead (DCLL).

CEA's nuclear analysis approach is based on the TRIPOLI- $4{ }^{\circledR}$ Monte Carlo code [5] and the JEFF-3.1.1 [6] nuclear data library when the other associations mainly use the MCNP5 Monte Carlo code [7] and the JEFF (3.2 or 3.1.1) or the FENDL (3 or 2.1) [8] libraries. To ensure consistency, these different approaches are compared. The nuclear analysis of DEMO HCLL carried out at ENEA [9] is compared to the CEA one. This comparison is not a rigorous benchmark between TRIPOLI- $4{ }^{\circledR}$ and MCNP5 since the nuclear data are different, this kind of exercise have been done for ITER [10].

This paper presents the comparison of relevant nuclear responses: neutron wall loading, tritium breeding ratio, nuclear heating, neutron flux distribution, etc. between CEA (TRIPOLI-4 ${ }^{\circledR}+$ JEFF-3.1.1) and ENEA (MCNP5+FENDL-2.1) neutronic analyses in DEMO
HCLL configuration. Section 2 describes the HCLL blanket design and section 3 the Monte Carlo models. The section 4 is devoted to results.

\section{HCLL blanket design}

The HCLL breeding blanket layout is a multi-module segment design. Modules are welded in a stiff poloidal back plate in order to form a banana-shaped segmentation (Fig. 1 left) that can be removed from the upper port. The back supporting structure (BSS) also works as a manifold, collecting and distributing lithiumlead and helium in the different blanket modules.
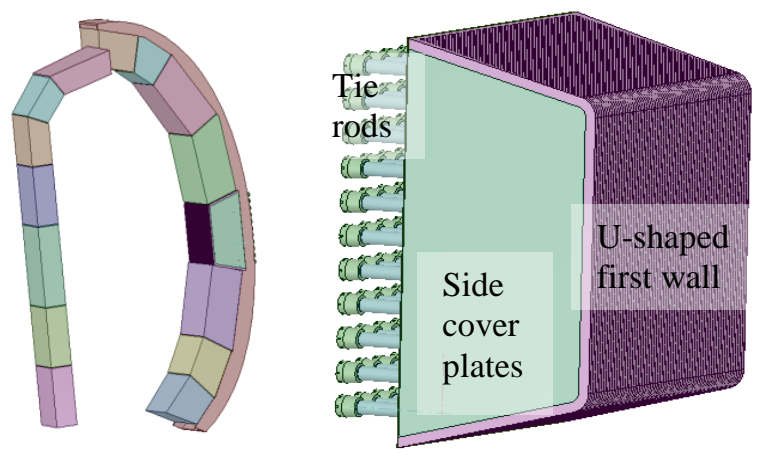

Fig. 1 HCLL DEMO segmentation (left) and equatorial outboard blanket module (right)

The design of outboard equatorial HCLL module is shown in Fig. 1 (right). Each HCLL blanket module consists of an Eurofer [11] steel box formed by an Ushaped plate composing the First, the upper and the bottom walls, closed on its sides by Side Cover plates and on the back by a set of Back Plates and tie rods (for BSS attachments).

The blanket module structure is reinforced by an inner grid of radial-poloidal and radial-toroidal 
Stiffening Plates (Fig. 2). The Stiffening Plates defines an array of internal cells where the Breeder Units are located. The eutectic $\mathrm{Pb}-\mathrm{Li}$ (enriched $90 \%$ in ${ }^{6} \mathrm{Li}$ ) flows around parallel horizontal Cooling Plates. An inlet and an outlet chamber on the Breeder Unit back plate ensure the helium distribution and collection for the Cooling Plates. All the plates, except the back plates constituting the manifolds, have internal cooling channels with a rectangular section.

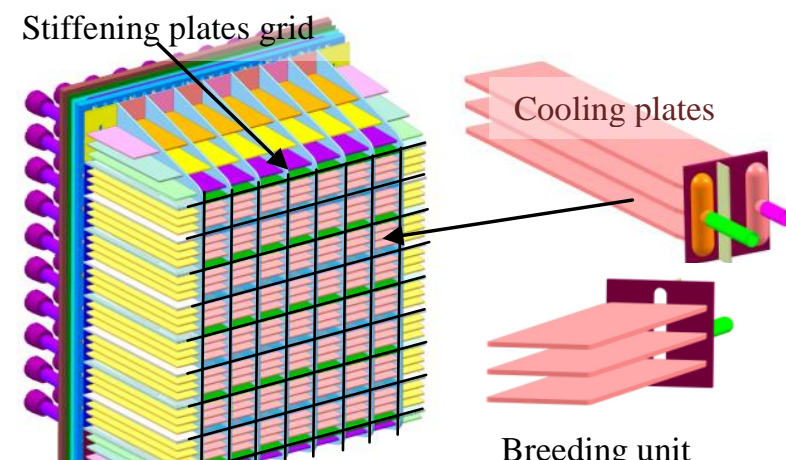

Fig. 2. Isometric view of the inner structure of the equatorial outboard blanket module (left) and detail of a single breeder unit (right)

\section{HCLL DEMO model}

This part describes the MCNP5 and TRIPOLI- $4^{\circledR}$ HCLL DEMO models in sub-section 3.1 and 3.2 respectively.

\subsection{MCNP5 model}

The MCNP5 model of DEMO HCLL was built at ENEA; it is based on a generic model generated by KIT using the McCad automated conversion tool [12]. The parameters of the studied tokamak are presented in Table 1. They correspond to a near-term DEMO, with conservative baseline design [13].

Table 1. Main parameters of the DEMO reactor.

\begin{tabular}{ll}
\hline Major radius, (m) & 9.0 \\
Minor radius, (m) & 2.25 \\
Plasma elongation & 1.66 \\
Plasma triangularity & 0.33 \\
Plasma peaking factor & 1.7 \\
Fusion power, (MW) & 1572. \\
Net electric power, (MW) & 500.0 \\
\hline
\end{tabular}

The segmentation has been implemented in the MCNP5 generic model using the MCAM CAD converter [14]. To ease CAD import only empty modules are considered. A single Outboard breeding unit (BU) has been converted by means of MCAM. Thus the obtained model has been replicated inside each blanket box to obtain a complete outboard segment using the repeated structure and rotation matrix feature of MCNP5 (FILL and LAT cards). The model of the inboard BU has been manually implemented and replicated in the inboard modules with the same technique used at the outboard. All the sub-components (including $\mathrm{Li}-\mathrm{Pb}$ and He manifolds, pipes and stiffening rods) up to the rear back-plate of the module are described in detail, while the back-supporting zone is represented as a homogeneous mixture of Eurofer, $\mathrm{He}$ and LiPb. Fig. 3 presents a poloidal-radial cut of the whole DEMO HCLL model. Fig. 4 shows the internal structure: stiffening grids, cooling plates, back plates and manifolds of the equatorial module.

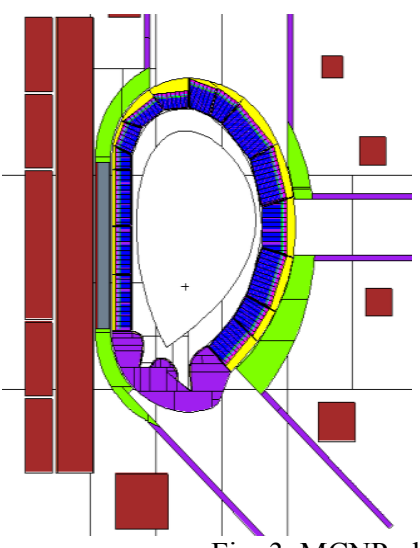

Color legend

- plasma chamber (white)

- breeder zone (blue),

- BSS (yellow)

- divertor (purple),

- vacuum vessel (green),

- port structures (purple)

- coils (brown).

For interpretation of the references to color, the reader is referred to the web version of this article

Fig. 3. MCNP plot of the DEMO HCLL model

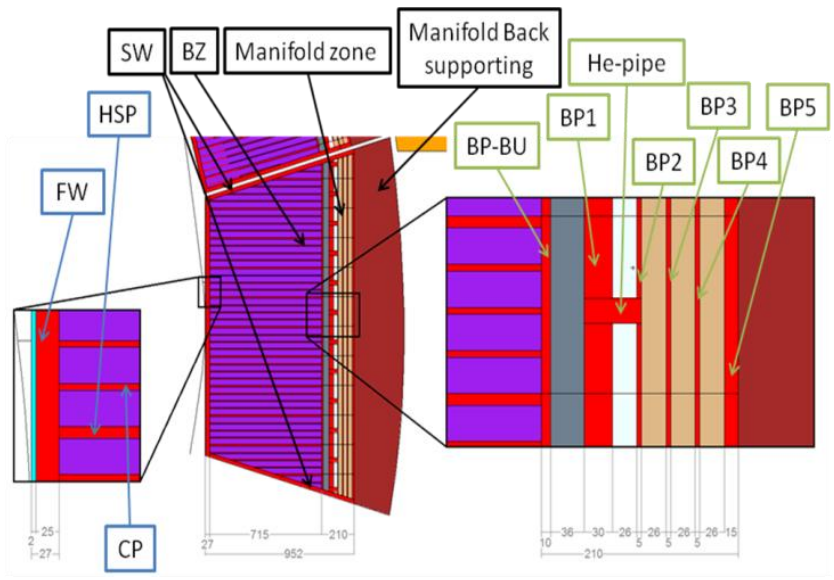

Fig. 4 MCNP model of HCLL Outboard module vertical section (dimensions are in $\mathrm{mm}$ )

\subsection{TRIPOLI-4 ${ }^{\circledR}$ model}

The DEMO generic model in TRIPOLI- $4{ }^{\circledR}$ format was generated by McCad [15]. The breeding blanket modules (BBM) segmentation, Breeder Unit geometry and compositions were defined using the MCNP5 model, kindly provided by ENEA.

Firstly the BBM were converted from MCNP5 input deck to TRIPOLI- $4^{\circledR}$ input file, using a python program that automates syntax conversion. Then BBM were implemented in the generic model. Inboard and Outboard BU were converted also and were duplicated in each BBM with an appropriate rotation transformation. TRIPOLI- $4^{\circledR}$ model BU rotation transformation are not exactly the same than the MCNP5 one, because we assume parallel conditions of BU's back plate and BBM's first wall surfaces. Nevertheless usually there are slight differences between both rotation transformations. The Fig. 6. Illustrate this difference in the upper inboard module. But most modules are identical between both models only the upper ones makes a distinction (BBM number 7-8-9 see Fig.7). 

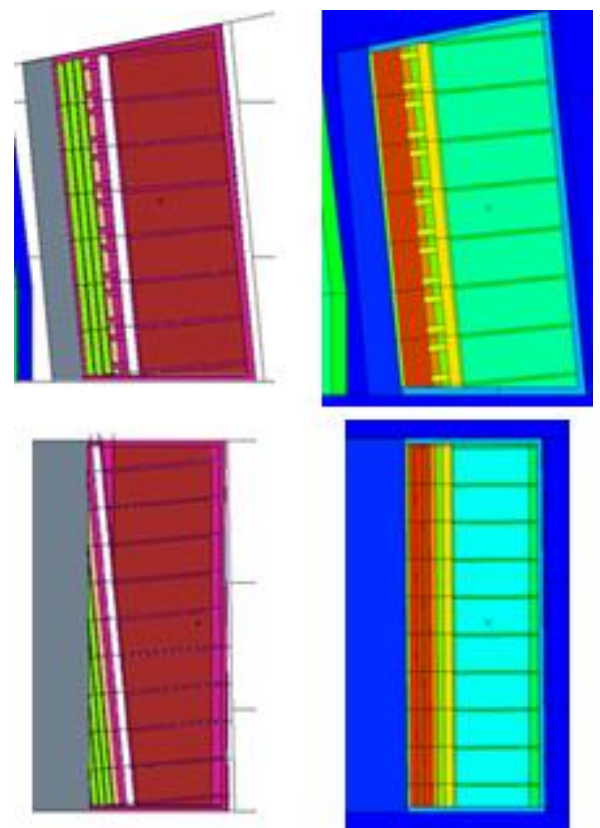

Fig. 6. BU rotation transformation comparisons between MCNP5 (left) and TRIPOLI- ${ }^{\circledR}$ (right) models; equatorial inboard module (top) upper inboard module (down)

Currently, there is no suitable rotation feature in TRIPOLI- $4^{\circledR}$ so a major part of the HCLL model building was devoted to the development of a python program that defines the rotation transformation as function of the BBM orientation and create the necessary surface and volume of the rotated BU. Discussions with the TRIPOLI $-4^{\circledR}$ Team are underway to define the proper development to be implemented to this end.

Finally, using the lattice functionality of TRIPOLI- $4^{\circledR}$ a complete DEMO HCLL model was obtained. BU is repeated in toroidal and poloidal direction in each BBM. Fig. 7 shows the TRIPOLI- $4^{\circledR}$ model.

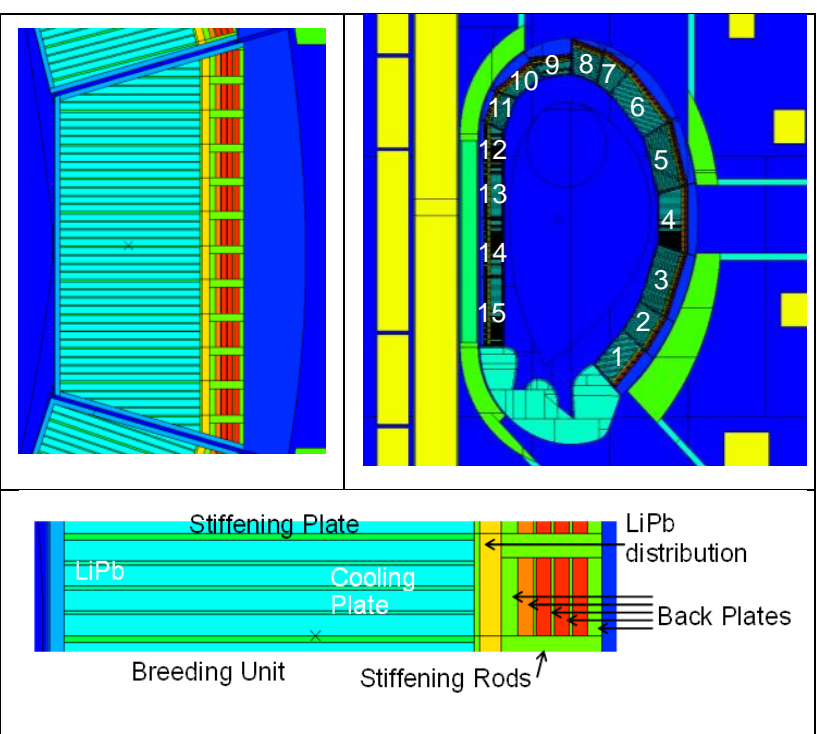

Fig. 7. TRIPOLI- $4^{\mathbb{R}}$ plots (poloidal-radial) of the DEMO HCLL: equatorial module (top left), DEMO and BBM (top right), breeding unit (bottom)
In this section neutron wall loading, tritium breeding ratio, nuclear heating and neutron flux distribution obtained at ENEA (MCNP5+FENDL-2.1) and CEA (TRIPOLI- ${ }^{\circledR}+$ JEFF-3.1.1) are compared. This exercise is not a rigorous benchmark between both codes since:

- The nuclear data are different, nevertheless the references [16] and [17] shows that JEFF-3.1.1 and FENDL-2.1 can be reasonably compared in fusion application.

- The geometries are slightly different in the upper modules (Fig 6).

\subsection{Neutron Wall loading}

First of all, the NWL calculated by TRIPOLI- $4{ }^{\circledR}$ was compared to the MCNP5 one. This comparison enables neutron sources verification; both models use the KIT FORTRAN subroutine describing the plasma neutron emission. NWL is defined by the neutron current (normalised to the fusion power) crossing the first wall surface divided by the first wall area; NWL is expressed in $\mathrm{MW} / \mathrm{m}^{2}$. To avoid the back scattering of neutrons in the current tallying (due to reflective surface) the neutrons must be killed after passing through the first wall. In MCNP5 the importance is set to 0 in all cells except the plasma chamber. In TRIPOLI- $4^{\circledR}$ the importance cannot be defined volume by volume. Its variance reduction technic is based on a mesh given by the user and the code automatically sets the importance in this mesh using various methods described in [5]. Another way was used to kill the neutrons crossing the first wall surface: setting leakage condition at these surfaces.

Fig. 8 shows the obtained poloidal NWL. It was estimated on each BBM first wall surfaces numbered 1 to 15 (see Fig. 7). There is a good agreement on the NWL calculated by each codes (nuclear data have not impact in NWL calculation). Discrepancies range from $1.6 \%$ to $2.8 \%$ with an averaged statistical error of $1 \%$ $(1 \sigma)$.

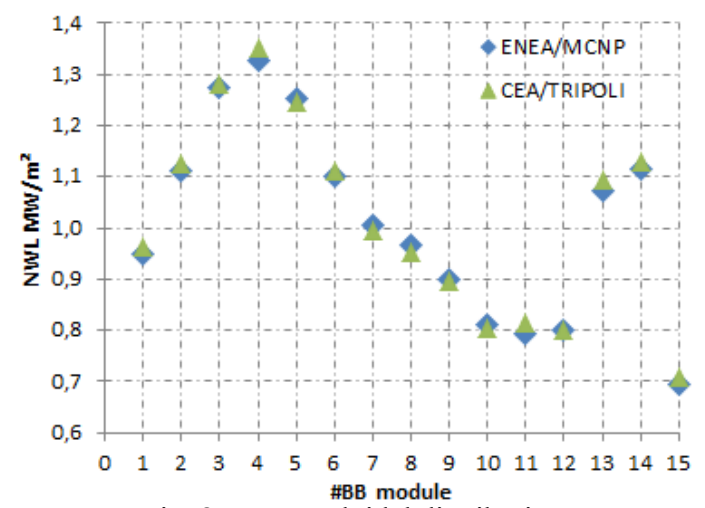

Fig. 8. NWL poloidal distribution

\subsection{Tritium breeding ratio}

There is no difference in TBR calculation methodology between both codes; ${ }^{6} \mathrm{Li}(\mathrm{n}, \mathrm{t})^{4} \mathrm{He}$ and ${ }^{7} \mathrm{Li}\left(\mathrm{n}, \mathrm{n}^{\prime}\right) \mathrm{T},{ }^{4} \mathrm{He}$ reaction rates are estimated and divided by the neutron source intensity. The overall TBR is estimated to 1.07 , it includes tritium production in the

\section{Results}


breeding zone, manifolds and BSS. This TBR is close to the ENEA one (1.06). There is also a good agreement in TBR poloidal distribution (TBR breakdown in each BBM) between both simulation; discrepancies ranges from $-3.6 \%$ to $2.1 \%$. Statistical errors $(1 \sigma)$ are around $0.1 \%$ for the overall TBR and $0.2 \%$ for the TBR BBM distribution. Differences in TBR are greater than the statistical errors; they are due to nuclear data library (JEFF-3.1.1 - FENDL-2.1) and geometry differences in upper BBM illustrated in Fig 6.

\subsection{Nuclear Heating}

Such as MCNP5's tally F6, TRIPOLI-4 ${ }^{\circledR}$ has specific estimators for the Nuclear Heating (NH) scoring based on the collision estimator and the energy balance. This estimator called DEPOSITED_ENERGY was completely rewritten in version 8 of the code released in 2013 [5]. A huge effort of code and nuclear data verification was carried out to ensure energy balance coherence (comparison with NJOY HEATR module). In recent benchmark comparisons, between MCNP5 \& TRIPOLI- $4^{\circledR}$, a good agreement is noticed for $\mathrm{NH}$ calculation: DEMO generic model [15] and ITER A-lite [10].

NH in DEMO HCLL components are reported in Table 2 for both Monte Carlo code simulations. A good agreement between both codes is observed, discrepancies range from $-4 \%$ to $3 \%$ with a statistical error $(1 \sigma)$ of maximum $1 \%$ in $\mathrm{VV}$. The total nuclear heating is used to calculate the energy multiplication $\left(\mathrm{M}_{\mathrm{E}}\right)$ factor, it is the ratio of the total nuclear power over the fusion neutron power ( $80 \%$ of FP). $\mathrm{M}_{\mathrm{E}}$ obtained with TRIPOLI- $4{ }^{\circledR}$ is equal to 1.19 not far $(-1.6 \%)$ from the MCNP5 one (1.21).

The poloidal $\mathrm{NH}$ distribution within each BBM of both simulations (ENEA \& CEA) presents a good agreement; discrepancies range from $-3 \%$ to $1 \%$. A statistical error $(1 \sigma)$ of $0.5 \%$ is achieved in each BBM.

The radial nuclear heating profiles across inboard mid-plane were compared, they are presented in Fig. 9. Variance reduction techniques were used in MCNP5 simulation to obtain results with reasonably low statistical errors up to the toroidal field coil region (lower than 20\%). Analog simulations were used in TRIPOLI- $4^{\circledR}$ with a large number of histories (140 billion) to achieve the same order of statistical error (around 20\%). This simulation will be used to validate, in a near future, variance reduction technique with TRIPOLI- $4^{\circledR}$ for coupled neutron photon simulations (in $\S 4.4$ TRIPOLI- $4^{\circledR}$ variance reduction technique application are showed for neutron simulation). Differences in $\mathrm{NH}$ (indicated hereafter in parenthesis) are: $\pm 4 \%$ in: first wall $\left(20 \mathrm{~W} / \mathrm{cm}^{3}\right)$, breeding zone $(6.3$ $\mathrm{W} / \mathrm{cm}^{3}$ in the first $5 \mathrm{~cm}$ ), manifold (around $0.3 \mathrm{~W} / \mathrm{cm}^{3}$ ) and vacuum vessel (maximum $0.9 \mathrm{~W} / \mathrm{cm}^{3}$ ), less than $20 \%$ in coils (around $90 \mathrm{~W} / \mathrm{m}^{3}$ ) but statistical error in this region is $20 \%(1 \sigma)$.

\begin{tabular}{llll}
\hline Components & $\begin{array}{l}\text { ENEA } \\
(\mathrm{MW})\end{array}$ & $\begin{array}{l}\text { CEA } \\
(\mathrm{MW})\end{array}$ & Discr. \\
\hline BBMs & 1216 & 1200 & $-1 \%$ \\
BSS & 24 & 25 & $3 \%$ \\
VV & 67 & 64 & $-4 \%$ \\
Divertor & 218 & 214 & $-2 \%$ \\
Total & 1527 & 1503 & $-2 \%$ \\
\hline
\end{tabular}

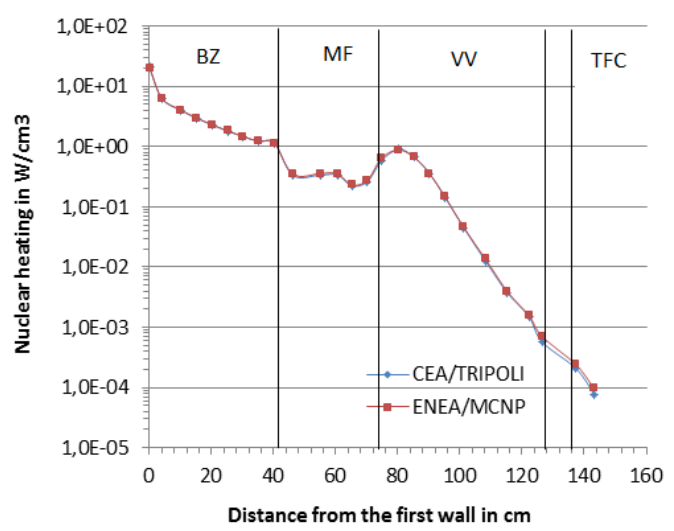

Fig. 9. Nuclear power density radial profile across inboard mid-plane

\subsection{Inboard radial profile of neutron flux, displacement damage and helium production}

The neutron flux, displacement damage rate and helium production have been calculated along the inboard mid-plane. The nuclear quantity is averaged on a poloidal height of $50 \mathrm{~cm}$ (from $\mathrm{z}=10$ to $\mathrm{z}=60 \mathrm{~mm}$ ). Variance reduction techniques have been used in both codes to obtain results with reasonably low statistical errors up to the toroidal field coil region (around 15\% in MCNP5 simulation and 5\% in TRIPOLI $-4^{\circledR}$ simulation). The mesh tallies feature of both codes has been applied to evaluate the neutron flux. Fig. 10, 11, 12 show respectively neutron flux, displacement damage and He production for both simulation. The results are consistent, discrepancies are: $\pm 5 \%$ in breeding zone and manifold, up to $10 \%$ in vacuum vessel and $16 \%$ in toroidal field coils but statistical error $(1 \sigma)$ in this region is around $15 \%$.

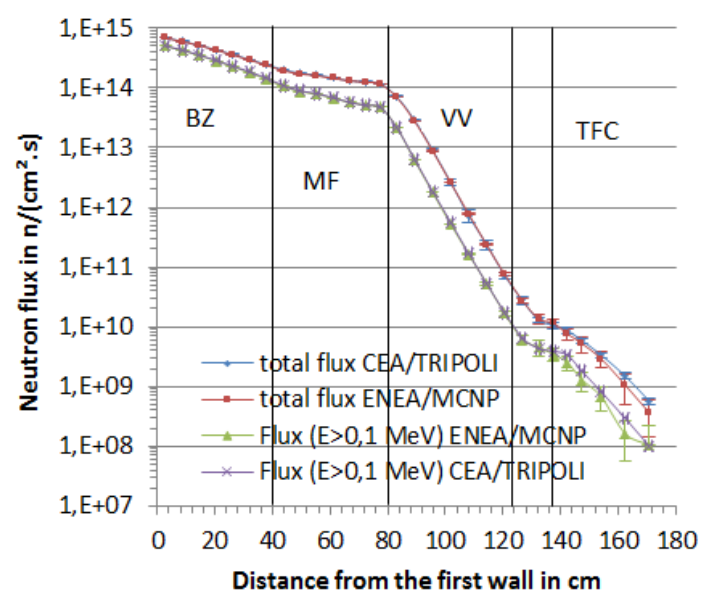

Fig. 10. Inboard radial neutron flux profile 


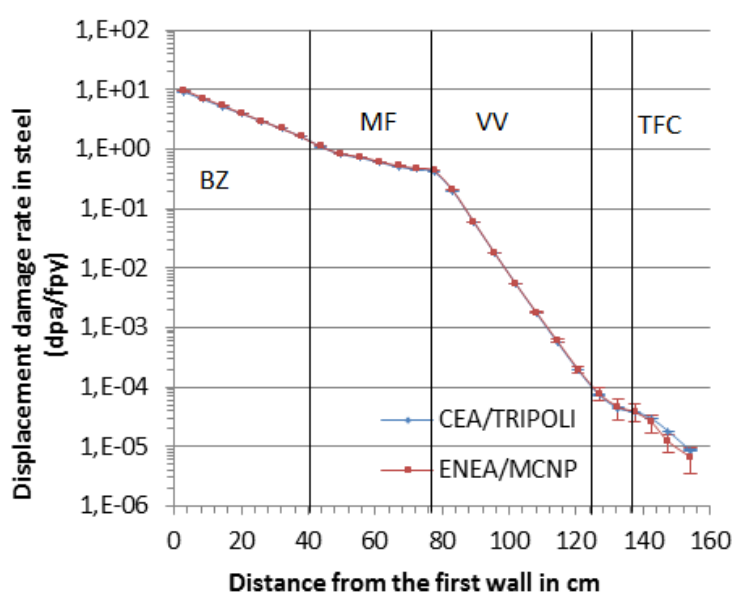

Fig. 11. Inboard radial displacement damage rate profile

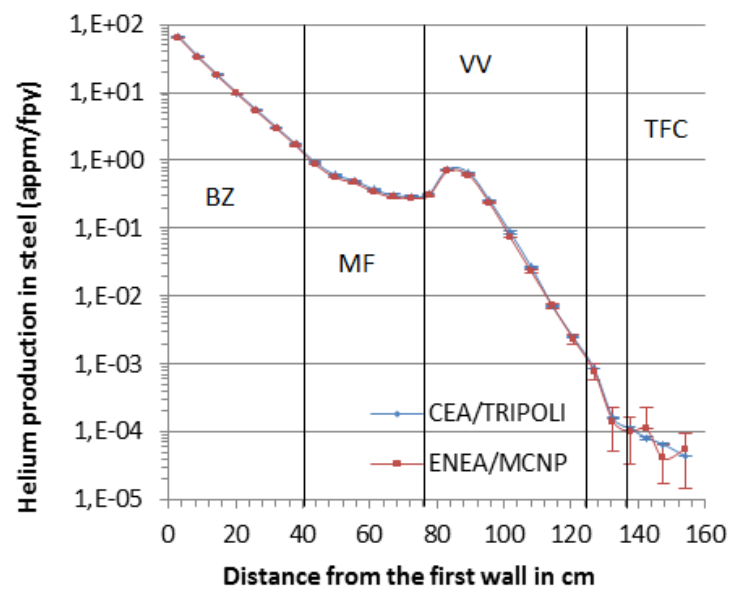

Fig. 12. Inboard radial helium production profile

Maximum values obtained in neutron flux (total and $\mathrm{E}>0.1 \mathrm{MeV})$, dpa and helium production are respectively: $6.9 \times 10^{14}, 5.0 \times 10^{14} \mathrm{n} /\left(\mathrm{cm}^{2} . \mathrm{s}\right), 9.5 \mathrm{dpa} / \mathrm{fpy}$, $65 \mathrm{appm} / \mathrm{fpy}$ in the first wall; $6.9 \times 10^{13}, 2.2 \times 10^{13}$ $\mathrm{n} /\left(\mathrm{cm}^{2} . \mathrm{s}\right), 0.2 \mathrm{dpa} / \mathrm{fpy}, 0.7 \mathrm{appm} / \mathrm{fpy}$ in the vacuum vessel and $8.0 \times 10^{9}, 3.0 \times 10^{9} \mathrm{n} /\left(\mathrm{cm}^{2} . \mathrm{s}\right), 2.7 \times 10^{-5} \mathrm{dpa} / \mathrm{fpy}$, $9.0 \times 10^{-5} \mathrm{appm} / \mathrm{fpy}$ in the toroidal field coil case.

Discrepancies in inboard radial profile ( $\mathrm{NH}$ included) between both nuclear analyses are within the statistical errors in the rear part: vacuum vessel and toroidal field coils. In the front part discrepancies are greater than the statistical error that is probably due to nuclear data difference and their treatment by each codes [18].

\section{Conclusions}

This paper proofs consistency of the nuclear analyses performed by ENEA and CEA on the HCLL DEMO using a different methodology with different calculation tools: MCNP5 Monte Carlo code and FENDL-2.1 nuclear data library at ENEA and TRIPOLI-4 ${ }^{\circledR}$ Monte Carlo code and JEFF-3.1.1 library at CEA. This consistency is a pre-condition for the application to DEMO nuclear analyses within the European breeder blanket program to ensure that results are comparable. The overall difference between CEA and ENEA nuclear analysis is around $\pm 2 \%$ in $\mathrm{TBR}$ and $\mathrm{M}_{\mathrm{E}}$, and $\pm 5 \%$ in neutron flux, nuclear heating, displacement damage and He production radial profile this is mainly due to nuclear data and their treatment by each code.

\section{Acknowledgments}

This work has been carried out within the framework of the EUROfusion Consortium and has received funding from the Euratom research and training programme 2014-2018 under grant agreement No 633053. The views and opinions expressed herein do not necessarily reflect those of the European Commission.

\section{References}

[1] https://www.euro-fusion.org/.

[2] http://ec.europa.eu/programmes/horizon2020/.

[3] L.V. Boccaccini et al., "Objectives and status of EUROfusion DEMO blanket studies”, ISFNT-12, 2015

[4] G. Aiello, J. Aubert, et al., Development of the Helium Cooled Lithium Lead blanket for DEMO, Fus. Eng. Des. Volume 89, Issues 7-8, October 2014, Pages 1444-1450.

[5] TRIPOLI-4 Project Team, TRIPOLI-4 version 8 User Guide, CEA-R-6316, Feb. 2013.

http://www.oecd-nea.org/tools/abstract/detail/nea-1716/.

[6] A. Santamarina et al., The JEFF-3.1.1 Nuclear Data Library JEFF, Report 22, 2009.

https://www.oecd-nea.org/dbdata/nds_jefreports/jefreport22/nea6807-jeff22.pdf.

[7] X-5 Monte Carlo Team, MCNP, LANL report LA-UR-031987, 2003.

[8] D. Lopez Aldama and A. Trkov, FENDL-2.1, Update of an evaluated nuclear data library for fusion applications, IAEA report INDC-NDS-467, 2004.

[9] R. Villari, WP13-SYS02-T06 Tritium breeding ratio assessment for HCLL DEMO, 2014. https://user.efda.org/?uid=2ME6F2

[10] J.-C. Jaboulay et al. "TRIPOLI- $4^{\circledR}$ Monte Carlo code ITER A-lite neutronic model validation,” Fus. Eng. Des. 89 (2014) 2174-2178

[11] B. Van der Schaaf, et al., The development of Eurofer reduced activation steel, Fus. Eng. Des. 69 (2003) 197203.

[12] L. Lu et al., Improved algorithms and advanced features of the CAD to MC conversion tool McCad, Fus. Eng. and Des. 89 (2014) 1885-1888.

[13] G. Federici et al., Overview of EU DEMO design and R\&D activities, Fus. Eng. Des. 89 (2014) 882-889.

[14] Y. Wu, FDS Team, CAD-based interface programs for fusion neutron transport simulation, Fus. Eng. Des. 84, 1987-1992, 2009.

[15] J.-C. Jaboulay et al., Monte Carlo tools Evaluation for Nuclear Analyses of the European DEMO, Fus. Eng. Des., In Press, 2015

[16] K. Kondo et al., Re-analysis of HCPB/HCLL Blanket Mock-up Experiments Using Recent Nuclear Data Libraries, Nuclear Data Sheets 120 (2014) 235-238

[17] C. Konno et al., Analyses of fusion integral benchmark experiments at JAEA/FNS with FENDL-2.1 and other recent nuclear data libraries, Fus. Eng. Des., 83 (2008) 1774-1781

[18] C. Jouanne, O petit, Impact of the interpolation mode on secondary particle energies for shielding and criticality benchmarks with TRIPOLI- $4^{\circledR}$ Monte Carlo Code, Proc. of Conf. PHYSOR 2014, Kyoto, japan, 28/9 - 3/10, 2014 